\title{
SWINGING UP THE FURUTA PENDULUM AND ITS STABILIZATION VIA MODEL PREDICTIVE CONTROL
}

\author{
Pavol Seman — Boris Rohal-Ilkiv — Martin Juhás — Michal Salaj
}

\begin{abstract}
This paper deals with certain options on controlling an inverted rotary pendulum also known as the Furuta pendulum. Controlling an inverted pendulum involves two stages. The first stage is the swing up of the pendulum and the second stage is its balancing in the up-right position. The paper describes two possibilities on swinging up the pendulum. First one is the classical approach based on comparing the current total (potential and kinetic) energy of the system with the energy in its stabilized up-right position. The second option uses an exponentiation operation over the pendulum position since the trend of power law function is very convenient for determining the amount of required energy to be delivered to the system. For the purposes of balancing the pendulum in the up-right position a predictive controller based on optimal control law with perturbation was proposed, which is an LQ controller with control signal corrections when constraints are exceeded. The results are illustrated by real-time experiments on a laboratory rotary inverted pendulum setup.
\end{abstract}

K e y w ords: furuta pendulum, swing-up, stabilization, predictive control, constraints

\section{INTRODUCTION}

The inverted pendulum is a great example of a nonlinear underactuated mechatronic system with fast dynamics and is therefore used for exploring various techniques of controlling such systems.

The inverted pendulum under the terms of rotary pendulum or furuta pendulum $[2,4]$ is a $3 \mathrm{D}$ device, Fig. 1, which means its movement is not planar as in the case of a cart-pendulum, but spatial (three-dimensional). The system has two degrees of freedom where on the first one exciting torque is applied. The device consists of two arms, one mounted horizontally and the other attached to it in a vertical direction representing the pendulum. This means that the rotary axes of both arms are perpendicular one to another. As already mentioned, different strategies of control can be explored using the inverted pendulum. These strategies can be divided into two categories. First are the swing up algorithms and the second category are the algorithms for balancing the pendulum. Swinging up the pendulum can be provided in many ways.

Possibly the simplest way is to swing up the pendulum with only one oscillation. The velocity of the exciting arm is set to maximum or any value high enough to place the pendulum in the horizontal position. After this is achieved (the velocity of the pendulum is near zero) the rotating arm changes its direction or stops. Once the pendulum comes near the equilibrium position due to inertia, the balancing controller takes over the control.

This method of swinging up is mentioned in eg [13]. The method requires minimum of time to realize, in that it places the pendulum to the vicinity of the up-right equilibrium position by applying only one oscillation. However this option is not ideal since an uncontrolled oscillation brings an unspecified amount of energy to the system and may cause the overtaking controller to fail in balancing the pendulum, leading to repeated attempts.

Certain opposite of the previous approach is a swing up with energy control. This method sequentially adds energy to the system by considering the energy of the pendulum in the up-right equilibrium position and actual energy [1]. Energy is supplied by oscillating movements of the exciting arm. This movement depends on the pendulum position and velocity. The main advantage of this strategy is that the system receives a specific amount of energy determined by the difference between the energy of the pendulum in the up-right position and actual energy. However, determining the control input is more difficult. A simple, reliable and effective compromise between the two mentioned approaches is the method of exponentiation of the pendulum position. Further explanations reveal the method of placing the pendulum effectively to the vicinity of the up-right position without oversupplying it with energy, hence not interfering with the process of balancing.

In this case balancing is achieved with the use of a predictive controller based on an LQ controller with corrections adjusting the control input when constraints are exceeded. The control algorithms presented in this paper were created in MATLAB Simulink and applied by means of $\mathrm{xPC}$ target.

\section{MATHEMATICAL MODEL}

The subject of this section is to briefly describe the mathematical model of the pendulum, Fig. 2, and its main

\footnotetext{
* Department of Automation, Measurement and Applied Informatics, Mechanical Engineering Faculty, Slovak University of Technology, Bratislava, boris.rohal-ilkiv@stuba.sk
} 


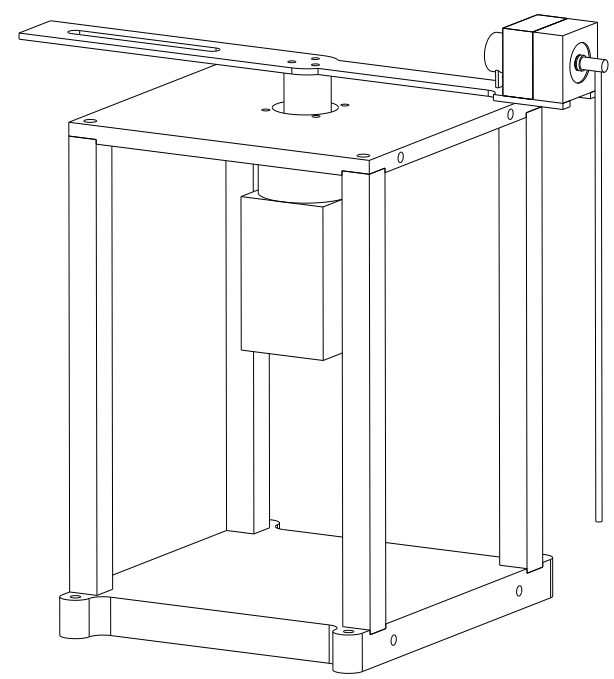

Fig. 1. Rotary inverted pendulum

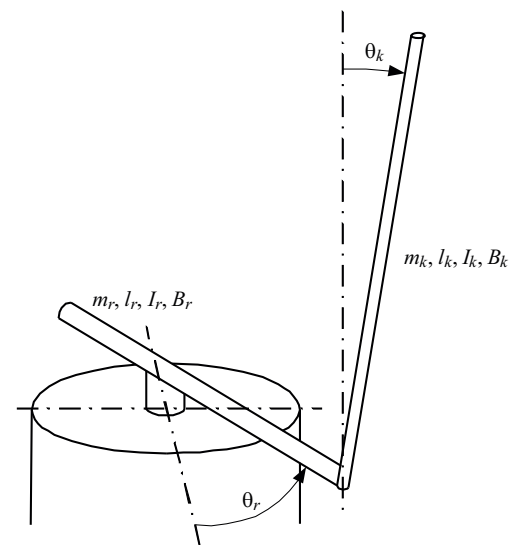

Fig. 2. Scheme of rotary inverted pendulum with base parameters

Table 1. Parameters of model

\begin{tabular}{lcc}
\hline Parameter & Symbol & Value \\
\hline Arm mass & $m_{r}$ & $0.63 \mathrm{~kg}$ \\
Arm length (from axis of & & \\
$\quad$ rotation to sensor) & $l_{r}$ & $0.22 \mathrm{~m}$ \\
Arm mass moment of inertia & $I_{r}$ & $0.021 \mathrm{~kg} \mathrm{~m}^{2}$ \\
Arm friction coefficient & $B_{r}$ & $0.08 \mathrm{~kg} \mathrm{~m}^{2} \mathrm{~s}^{-1}$ \\
Pendulum mass & $m_{k}$ & $0.062 \mathrm{~kg}^{-}$ \\
Distance from pendulum center & & \\
$\quad$ of mass to rotation axis & $l_{k}$ & $0.2 \mathrm{~m}$ \\
Pendulum mass $\quad$ moment of inertia & $I_{k}$ & $0.0012 \mathrm{~kg} \mathrm{~m}^{2}$ \\
Pendulum friction coefficient & $B_{k}$ & $0.0001 \mathrm{~kg} \mathrm{~m}^{2} \mathrm{~s}^{-1}$ \\
Pendulum position & $\theta_{k}$ & $\mathrm{rad}$ \\
Arm position & $\theta_{r}$ & $\mathrm{rad}$ \\
Torque (control action) & $\tau$ & $\mathrm{N} \mathrm{m}$ \\
\hline
\end{tabular}

parameters, Tab. 1, estimated for the exploited laboratory setup. More details concerning the laboratory setup and its identification can by found in [17]. Obtaining the model is necessary for realizing predictive balancing control.

The analytical model is based on the equations of motion derived from the Lagrange equations of the second kind, which represent the most commonly used method for establishing equations of motion of difficult mechanical systems. This method is applied for obtaining mathematical models of eg manipulators, with several degreases of freedom $[5,14]$. Taking into account damping in the form of friction, the resulting equations of motion of the pendulum are $[17]$

$$
\begin{gathered}
\ddot{\theta}_{r}=\left\{B_{k} m_{k} \cos \theta_{k} l_{k} l_{r} \dot{\theta}_{k}-B_{r} m_{k} l_{k}^{2} \dot{\theta}_{r}+\right. \\
\left(m_{k}^{2} l_{k}^{4} \dot{\theta}_{r}+m_{k} l_{k}^{2} I_{k} \dot{\theta}_{r}\right) \sin \left(2 \theta_{k}\right) \dot{\theta}_{k}+\left[\left(m_{k}^{2} l_{k}^{3} l_{r}+m_{k} l_{k} l_{r} I_{k}\right) \dot{\theta}_{k}^{2}\right. \\
\left.-m_{k}^{2} l_{k}^{3} l_{r} \cos ^{2} \theta_{k} \dot{\theta}_{k}^{2}-m_{k}^{2} g l_{k}^{2} l_{r} \cos \theta_{k}\right] \sin \theta_{k}-B_{r} I_{k} \dot{\theta}_{r}+ \\
\left.\left(m_{k} l_{k}^{2}+I_{k}\right) \tau\right\} /\left\{\left(l_{r}^{2} I_{k}+l_{k}^{2} I_{r}\right) m_{k}+\left(m_{k}^{2} l_{k}^{4}+m_{k} l_{k}^{2} I_{k}\right) \sin ^{2} \theta_{k}\right. \\
\left.-m_{k}^{2} l_{k}^{2} l_{r}^{2} \cos ^{2} \theta_{k}+m_{k}^{2} l_{k}^{2} l_{r}^{2}+I_{r} I_{k}\right\}, \quad(1) \\
\ddot{\theta}_{k}=-\left\{-m_{k}^{2} \sin \left(2 \theta_{k}\right) \cos \theta_{k} l_{r} l_{k}^{3} \dot{\theta}_{r} \dot{\theta}_{k}\right. \\
-\left(m_{k}^{2} \cos \theta_{k} l_{k}^{4} \dot{\theta}_{r}^{2}+m_{k}^{2} g l_{k}^{3}\right) \sin ^{3} \theta_{k}-B_{r} m_{k} \cos \theta_{k} l_{k} l_{r} \dot{\theta}_{r} \\
+\left(B_{k} m_{k} l_{r}^{2}+B_{k} I_{r}\right) \dot{\theta}_{k}+B_{k} m_{k} \sin ^{2} \theta_{k} l_{k}^{2} \dot{\theta}_{k}+m_{k} \cos \theta_{k} l_{k} l_{r} \tau \\
+\left[\left(m_{k}^{2} l_{r}^{2} l_{k}^{2} \dot{\theta}_{k}^{2}-m_{k}^{2} l_{r}^{2} l_{k}^{2} \dot{\theta}_{r}^{2}-m_{k} l_{k}^{2} I_{r} \dot{\theta}_{r}^{2}\right) \cos \theta_{k}-m_{k}^{2} g l_{k} l_{r}^{2}-\right. \\
\left.\left.m_{k} g l_{k} I_{r}\right] \sin \theta_{k}\right\} /\left\{\left(m_{k}^{2} l_{k}^{4}+m_{k} l_{k}^{2} I_{k}\right) \sin ^{2} \theta_{k}+\right. \\
\left.\left(l_{r}^{2} I_{k}+l_{k}^{2} I_{r}\right) m_{k}+I_{k} I_{r}-m_{k}^{2} \cos ^{2} \theta_{k} l_{k}^{2} l_{r}^{2}+m_{k}^{2} k_{k}^{2} l_{r}^{2}\right\} . \quad(2)
\end{gathered}
$$

These equations are further utilized to establish the state space model for generating the balancing algorithms [17]. The obtained model was then used in all experiments listed further in this paper.

\section{SWING-UP}

This topic introduces two possibilities of swinging up the pendulum to the vicinity of the up-right position. A traditional approach is swinging-up with energy control whereas the second method determines the control input by exponentiation of the pendulum position.

\subsection{Swing-up by energy control}

It is convenient when applying the strategy of sequential energy adding to consider the energy in the up-right position and the actual energy of the pendulum [1]. Energy is added by oscillating movements of the exciting arm. The movement of the arm relies on the position and velocity of the pendulum. Normalized actual energy is determined by

$$
E=\frac{m_{k} g l_{k}}{2}\left(\left(\frac{\dot{\theta_{k}}}{\omega_{0}}\right)^{2}+\cos \theta_{k}-1\right)
$$



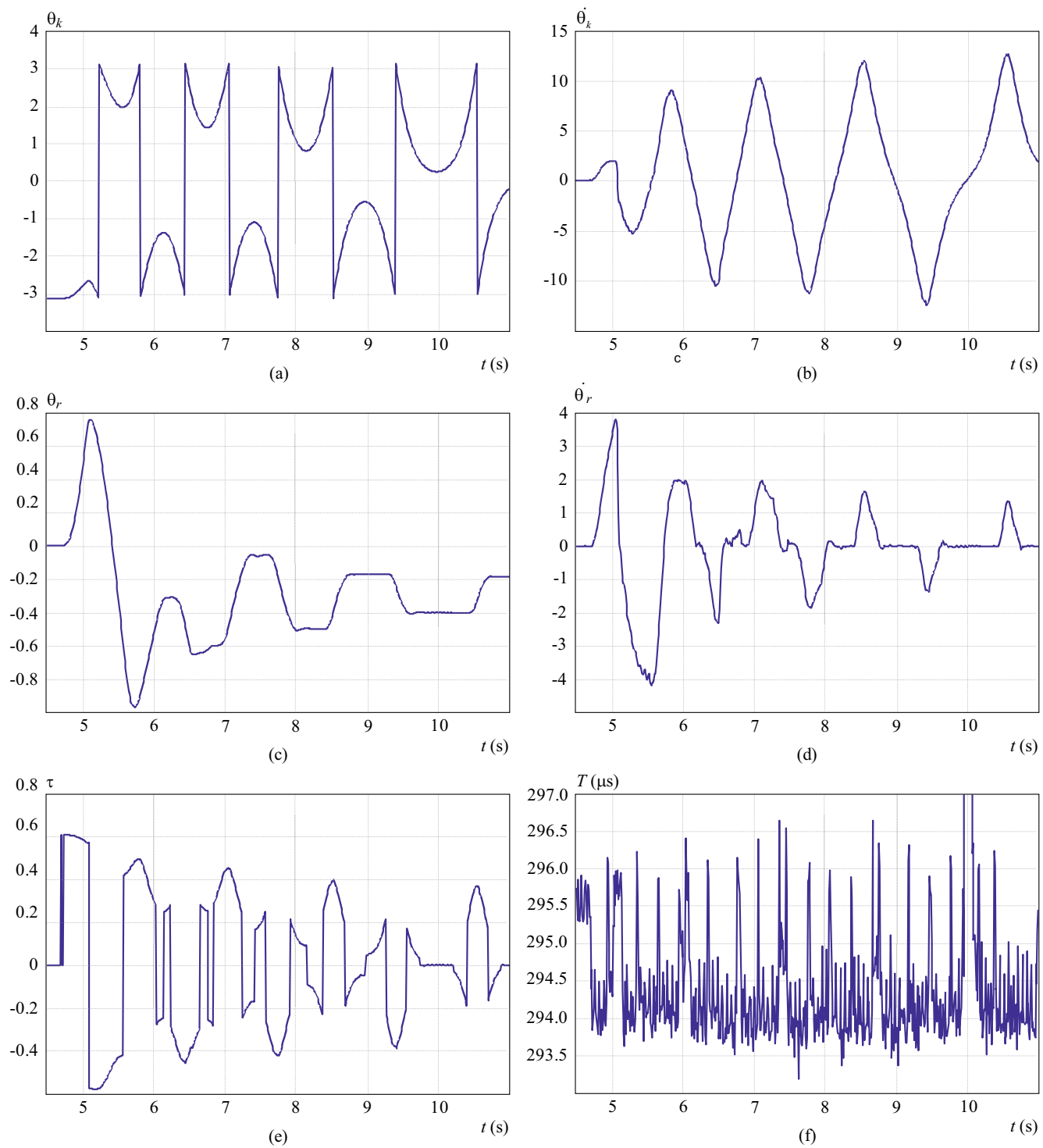

Fig. 3. Selected variables in swing-up by energy control: (a) - pendulum position, (b) - pendulum velocity, (c) — arm position, (d) - arm velocity, (e) - torque, (f) - computation time in one cycle

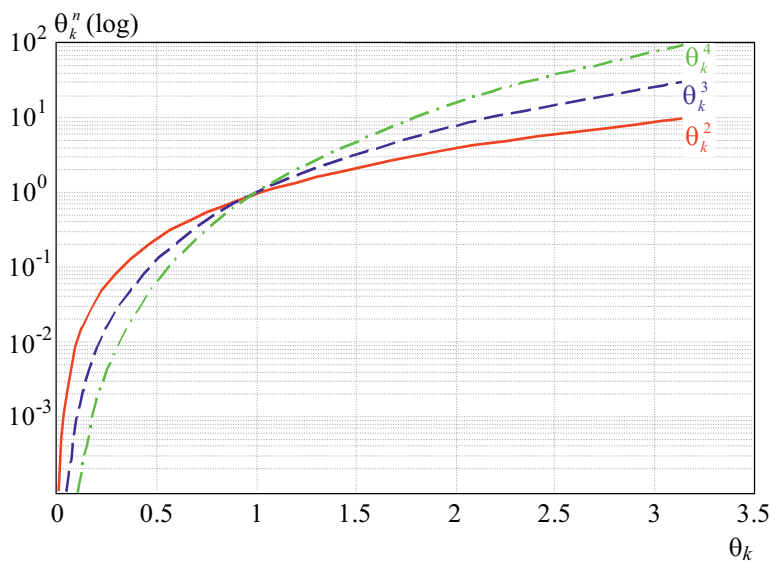

Fig. 4. Exponentiation of the pendulum position

where $\omega_{0}$ is the frequency of small fluctuations of the pendulum in the vicinity of the downward position.

$$
\omega_{0}=\sqrt{\frac{m_{k} g l_{k}}{I_{k}}}
$$

The value of the total energy depends on the selection of the "base" with zero energy. There are more options for the base: downward equilibrium position, horizontal position or up-right equilibrium position. The most suitable choice is the up-right equilibrium, since there is no reason to add more energy to the pendulum in this position. According to (3) the energy of the system in the downward position is $-2 m_{g} l_{k}$ and it is also the energy that needs to be delivered to the system. Therefore, it is necessary to apply a control law that would consider the current gained energy of the system and would at the same time add energy in the right direction

$$
u=\operatorname{sat}\left(k_{v}\left(E-E_{0}\right)\right) \operatorname{sign}\left(\dot{\theta}_{k} \cos \theta_{k}\right) .
$$

This is the role of the last member of the equation (5) $\operatorname{sign}\left(\dot{\theta_{k}} \cos \theta_{k}\right)$, where the pendulum velocity $\dot{\theta_{k}}$ defines the direction of movement and $\cos \theta_{k}$ indicates the position of the pendulum in the upper or lower half-plane. This polarity is the key in determining whether to "push" 

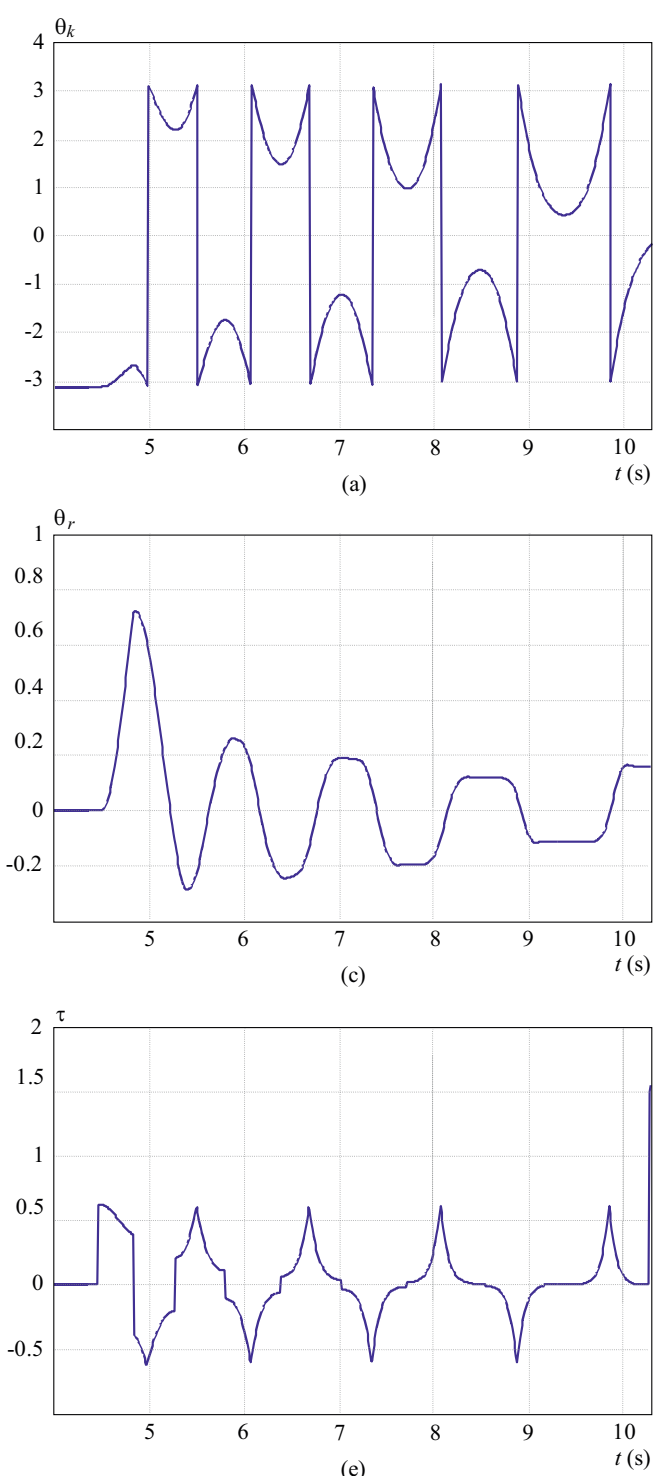

(e)

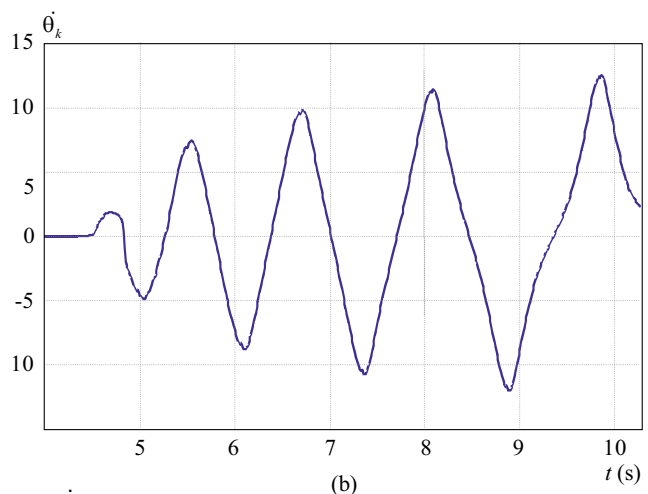

$\theta_{r}$
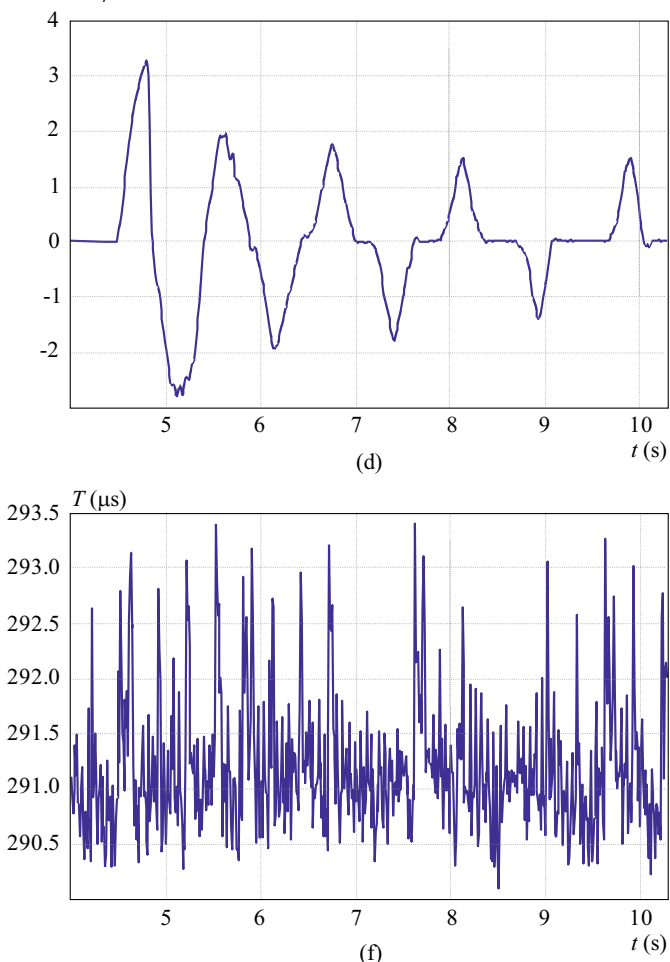

Fig. 5. Selected variables in swing-up by exponentiation of the pendulum position: (a) — pendulum position, (b) — pendulum velocity, (c) - arm position, (d) - arm velocity, (e) - torque, (f) - computation time in one cycle

or "pull" onto the mounted end of the pendulum. The first member of (5) specifies the required torque, where $E_{0}$ is the desired energy in the up-right unstable equilibrium position. As mentioned above, it is reasonable to consider it to be zero. The significance of the coefficient $k_{v}$ is to determine how fast the pendulum reaches the vicinity of the unstable equilibrium position. Saturation limits the value of the excitation torque. This way the energy is supplied to the pendulum by taking into account the energy of the pendulum in the up-right equilibrium position that characterizes a stable pendulum.

Swing up measurement results using the mentioned algorithm are shown in Fig. 3. Figure 3(a) shows the position in course of time. As one can see, the pendulum gradually approaches the up-right equilibrium position. At the end of the progress the pendulum is located in the up-right position where the control is taken over by the balancing controller. Figure $3(\mathrm{f})$ shows the computational demands of the algorithm. They are represented as the time necessary for computation in one cycle.

\subsection{Swing up by exponentiation of the pendu- lum position}

In this case the amount of supplied energy is dependent on the pendulum position. In view of the preferred progress of the exponential function, it is possible to supply the system with more energy when the pendulum is in the vicinity of the downward equilibrium position and less energy when the pendulum is in the vicinity of the up-right equilibrium position. The control law is simple

$$
u=k_{v}\left|\theta_{k}^{n}\right| \operatorname{sign}\left(\dot{\theta_{k}} \cos \theta_{k}\right)
$$

where $k_{v}$ and $n$ are constants, that are used to influence the dynamics of the pendulum swing up and $\theta_{k}$ is the position of the pendulum. Since the up-right position of the pendulum is set to $\theta_{k}=0$, the dynamics of the swing up is influenced by a suitable exponentiation of the position $\theta_{k}^{n}$, so that the amount of supplied energy is minimal as long as the pendulum is relatively near the vicinity of the upright position. This argument is founded 


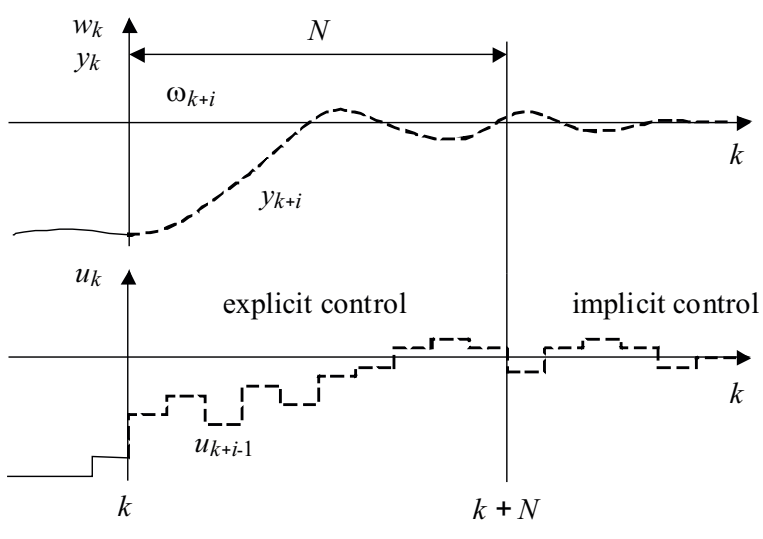

Fig. 6. Dual mode control law

on the progress shown in Fig. 4. That way it is possible to modify the amount of energy to be delivered to the system, depending on the pendulum position and thus secure adding only a small amount of energy when the pendulum is in the vicinity of the up-right position, thereby making the transition to balancing control smoother. In this case the position of the pendulum is in radians but can be converted to a different range, allowing to achieve the desired position requiring significantly less delivered energy.

Verification of functionality of the mentioned algorithm can be shown in Fig. 5. Constants used in the swing up were: $k_{v}=0.02$ and $n=3$. As seen from the progress of the pendulum position, Fig. 5(a), the pendulum is gradually approaching the up-right equilibrium position, where near this position the balancing algorithm can take over the control. The approaching is sequential and similar quality as in the swing up by energy control. Figure 5(f) shows the computation demands of the algorithm, which are similar or slightly less demanding than in the previous case. The advantage here is, that there are no pre-computations necessary as in the case of a swing up with energy control, where it is important to know the energy of the pendulum in the up-right position and the frequency of small pendulum oscillations.

\subsection{Balancing the pendulum using a predictive controller}

This method uses the mathematical model of the system. It was obtained using linearization of the equations of motion (1) and (2) in the upper equilibrium position [17]. Due to better conditionality for numerical calculation with model predictions, the technique of closed-loop paradigm [9] was employed. The model then is used to determine the sequence of control actions on a certain prediction horizon. Subsequently, only the first member of the sequence is applied. The prediction horizon is determined from the behavior of the model. A too short horizon leads to an unstable control whereas a too long horizon requires high computation demands. The horizon of prediction can also be infinite or finite. In this case the so-called dual mode control was used, see Fig. 6 [16]. Basically it is a combination of both finite and infinite horizon control, where the finite horizon control uses $N$ predicted steps (first mode of control) and leads the system to the desired invariant and feasible target set. Sequentially a closed-loop balancing controller is used to balance the system in the vicinity of the zero state (second mode of control) [7].

The control law is then

$$
u_{k+i}= \begin{cases}K x_{k+i}+c_{k+i} & \text { for } i=0, \ldots, N-1, \\ K x_{k+i} & \text { for } i \geq N\end{cases}
$$

where $K$ is the gain of the state balancing controller $[11,12]$ and $c_{k}$ are so-called perturbations [10] that secure feasibility of constraints along the finite horizon $N$. The cost function is

$$
J=\sum_{i=1}^{\infty}\left(x_{k+i}^{T} Q x_{k+i}+u_{k+i}^{T} R u_{k+i}\right)
$$

defined for an infinite horizon, but can be rewritten as

$$
J=\sum_{i=1}^{N-1}\left(x_{k+i}^{T} Q x_{k+i}+u_{k+i}^{T} R u_{k+i}\right)+x_{k+N}^{T} P x_{k+N}
$$

where penalization of the final state is added to the finite horizon cost function. Using the so-called autonomous augmented state-space description [8] the cost function is

$$
J=x_{k}^{T} P x_{k}+f_{k}^{T} S f_{k}
$$

where $f_{k}$ is vector of perturbations $c_{i}, i=1, \ldots N-1$. The value of $x_{k}^{T} P x_{k}$ is a function of the actual state $x_{k}$ and thereby for cost optimization with respect to $f_{k}$ insignificant. Therefore, it is sufficient to minimize the cost function in the form

$$
J=f_{k}^{T} S f_{k}+\text { const } .
$$

For solving this optimization problem, following constraining conditions were taken into account

- control action limitation

- achieving desired set $[3,18]$ at the end of the prediction horizon

The minimization of the cost function (11) was solved using a quadratic programming function and qpOASES software [6].

Figure 7 shows the progress of state variables and other quantities that are important in the process of swinging up and subsequent balancing of the pendulum. There is thus possible to observe the complex control of the rotary inverted pendulum, when the pendulum is swung up from the downward equilibrium position to the vicinity of the upright equilibrium position, where the control is taken over by the balancing controller.

The swing up was realized using the algorithm based on the exponentiation of the pendulum position with parameters: $k_{v}=0.02$ and $n=3$ and the above mentioned predictive controller with a prediction horizon of 

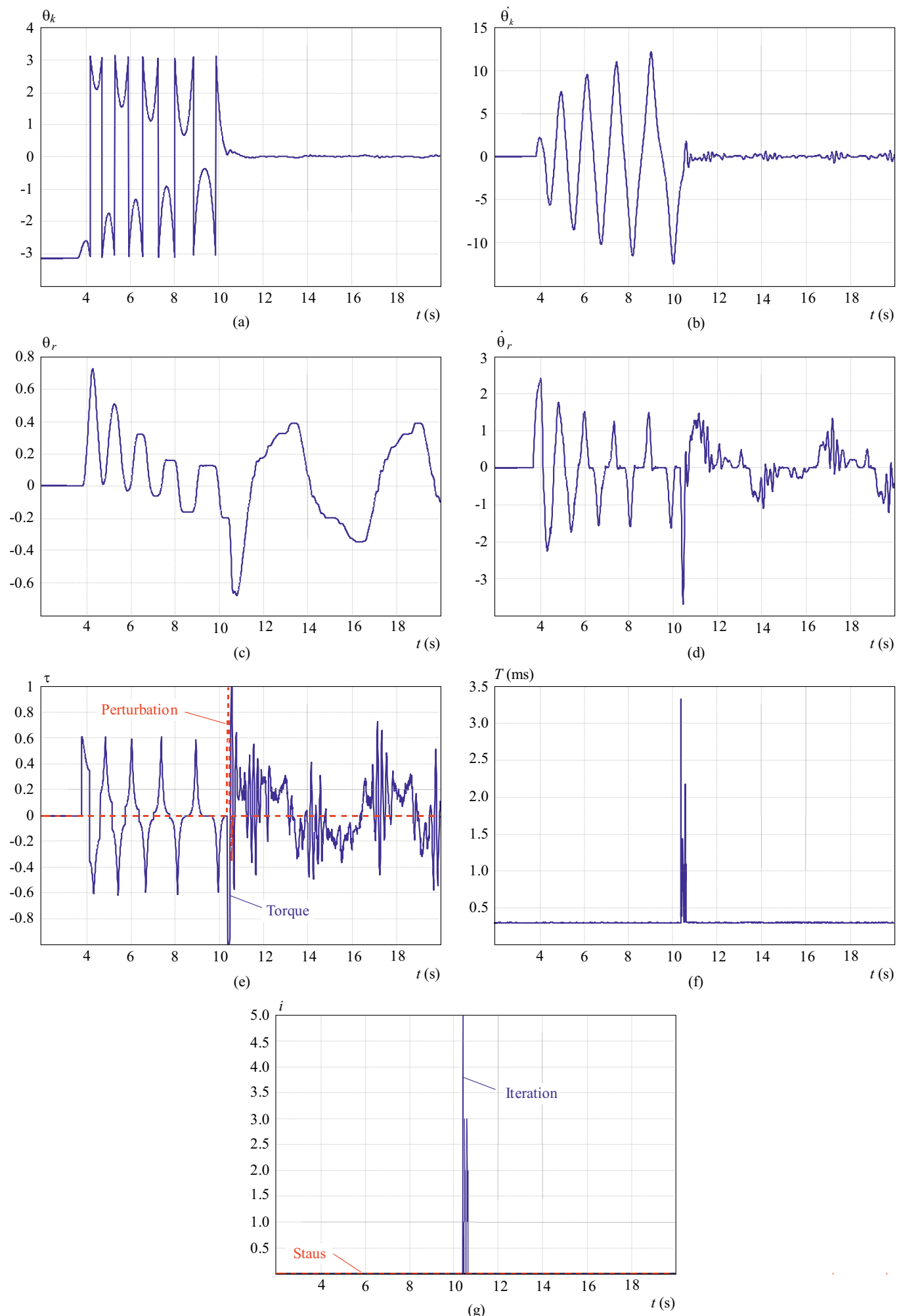

Fig. 7. Selected variables in the swing up based on the exponentiation of pendulum position and balancing using predictive controller: (a) - pendulum position, (b) - pendulum velocity, (c) - arm position, (d) - arm velocity, (e) — torque and perturbation, (f) computation time in one cycle, (g) - iterations and status

$N=100$ steps and control action limitation $\pm 1 N m$ was applied for balancing the pendulum. Position of the pendulum. Figure 7 (a) shows that the designed algorithm is capable of performing this task. In Fig. 7(e) one can follow the course of the torque, by which the pendulum is controlled. As it can be seen, the torque does not exceed specified limit.

Limitations were insured by perturbations that adjusted the control actions if constraints were exceeded. In Fig. 7(e) we can see, that when transiting from swing 
up to balancing, the constraints were active. In Fig. 7(f) we can observe an increase in computation time by active constraints and with the use of qpOASES. Since the issue is to find a numerical solution of a quadratic optimization problem, qpOASES uses a certain amount of iterations to find the suitable solution, which is shown in Fig. $7(\mathrm{~g})$. One can also see the status that informs whether a suitable solution was found ( 0 = found suitable solution).

\section{CONCLUSION}

The problem of swinging up the inverted rotary pendulum together with the model predictive balancing control was investigated. Two possibilities on swinging up the pendulum were described and tested on real laboratory setup. The first one was classical approach based on the energy of system in its stabilized up-right position, the second has used the exponentiation operation over the pendulum position for determining the amount of required energy to be delivered to the system. The paper presents the second option for swinging up the inverted pendulum, which is in compare with the method of energy control simpler but with similar results. For the purposes of balancing the pendulum in the up-right position a model based predictive controller with constrained optimal control law was used. The overall performance was illustrated by experiments with the real laboratory setup. The obtained results confirms the possibility of applying the predictive controller in similar mechatronic applications with fast dynamics.

\section{Acknowledgments}

The work has been supported by the Slovak Research and Development Agency under grants APVV-0280-06 and APVV-0090-10. This support is gratefully acknowledged.

\section{REFERENCES}

[1] ASTRÖM, H.-FURUTA, K. : Swing up a Pendulum by Energy Control, 13th world congress IFAC, San Francisco.

[2] AWTAR, S.-KING, N.: Inverted Pendulum Systems: Rotary and Arm-Driven - a Mechatronic System Design Case Study, Mechatronics 12 No. 2, 357-370.

[3] BLANCHINI, F.: Set Invariance in Control, Automatica 35 No. $11,1747-1767$.

[4] CHYE, T. K.-SANG, T. C. : Rotary Inverted Pendulum, 1999, A Final Year Project Report, School of electrical and electronic engineering, Nanyang Technological University.

[5] FANTONI, I.-LOZANO, R. : Non-Linear Control for Underactuated Mechanical Systems, Springer, London, 2002.

[6] FERREAU, H.: qpoases - Online Active Set Strategy, 2011, http://www.kuleuven.be/optec/software/qpOASES.

[7] KARAS, A.-ROHAĽ-ILKIV, B.-BELAVÝ, C.: Praktické aspekty prediktívneho riadenia, Slovenská technická univerzita v Bratislave, 2007. (in Slovak)
[8] KOUVARITAKIS, B.-LEE, Y.-TORTORA, G.-CANNON, M. : Mpc Stability Constraints and their Implementations, In: Preprints from the IFAC Conference Control System Design ( Kozák and M. Huba, eds.), Slovak University of Technology, Bratislava, Slovakia, 205-212.

[9] KOUVARITAKIS, B.-ROSSITER, J. A.-CHANG, A. O. T. : Stable Generalized Predictive Control: An Algorithm with Guaranteed Stability, IEE Proceedings Part D 139 No. 4 (1992), 349-362.

[10] KOUVARITAKIS, B.-ROSSITER, J.-SCHUURMANS, J. : Efficient Robust Predictive Control, Automatic Control, IEEE Transactions on 45 No. 8 (2000), 1545-1549, doi: 10.1109/9.871769.

[11] KWAKERNAAK, H.-SIVAN, R.: Linear Optimal Control Systems, Wiley-Interscience, 1972.

[12] LIN, C. F. : Advanced Control Systems Design, Prentice Hall, 1993.

[13] MERTL, J.-SOBOTA, J.: Swing-up and Stabilization of Rotary Inverted Pendulum, In 15. medzinárodná konferencia Process Control, 2005, volume 15, Štrbské Pleso.

[14] NOSKIEVIČ, P.: Modelování a identifikace systémů, Montanex, Ostrava, 1999.

[15] RAKOVIC, S.-MAYNE, D.: Set Robust Control Invariance for Linear Discrete Time Systems, In 44th IEEE Conference on Decision and Control, and the European Control Conference 2005, Seville, Spain, 2005, pp. 975-980.

[16] ROSSITER, J.-KOUVARITAKIS, B.-RICE, M. : A Numerically Robust State-Space Approach to Stable-Predictive Control Strategies, Automatica 34 No. 1 (1998), 65-73.

[17] SEMAN, P.: Predictive Control of Nonlinear Mechatronics Systems, 2012, PhD thesis, STU in Bratislava, Slovakia. (in Slovak)

Received 23 March 2012

Pavol Seman (Ing) is PhD student with the Institute of Automation, Measurement and Applied Informatics of Mechanical Engineering Faculty, STU in Bratislava. His research interests include MPC, modeling and control of mechanical underactuated systems.

Boris Rohal'-Ilkiv (Prof, Ing, CSc) is with the Institute of Automation, Measurement and Applied Informatics of Mechanical Engineering Faculty, STU in Bratislava. His research is mainly focused on identification, modeling, adaptive and predictive control of industrial processes and mechatronic systems, including combustion engines, mechanical underactuated systems and active vibration systems.

Martin Juhás (Ing, $\mathrm{PhD}$ ) is assistant professor with the Institute of Automation, Measurement and Applied Informatics of Mechanical Engineering Faculty, STU in Bratislava. His research interests include modeling and control of industrial processes and design of hardware and software tools for control of industrial systems.

Michal Salaj (Ing) is PhD student with the Institute of Automation, Measurement and Applied Informatics of Mechanical Engineering Faculty, STU in Bratislava. His research interests include nonlinear MPC and the processes state and parameter estimation. 